\title{
A Comparison between Two Corrected Geomagnetic Coordinate Systems at High-Latitudes
}

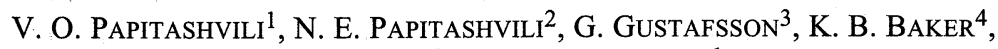 \\ A. RODGER ${ }^{5}$, and L. I. GROMOVA ${ }^{1}$ \\ ${ }^{1}$ IZMIRAN, Troitsk, Moscow Region, 142092, Russia \\ ${ }^{2}$ World Data Center B2, Moscow, 117296, Russia \\ ${ }^{3}$ Swedish Institute of Space Physics, Uppsala, S-75591, Sweden \\ ${ }^{4}$ Applied Physics Laboratory, JHU, Laurel, MD 20707, U.S.A. \\ ${ }^{5}$ British Antarctic Survey, Cambridge CB3 OET, England
}

(Received November 11, 1991; Accepted May 8, 1992)

\begin{abstract}
The existing corrected geomagnetic coordinate system is based on internal sources of the Earth's magnetic field and describes observed phenomena in a time-independent way. Space experiments, however, use universal time to follow the dynamics of observed phenomena in 3-dimensional space and external sources play an important role there. A new corrected coordinate system based on the "realistic" geomagnetic equator is proposed to order observations of experiments at each moment of time. The system is based on constant $B$-minimum ovals at the 3-D geomagnetic equator plane. The magnetospheric model by TSYGANENKo (1989) is used and an algorithm has been developed to derive the lines of constant latitude at ionospheric heights for a specific universal time. Combining these latitudes with corrected geomagnetic longitudes provides an opportunity to order polar ionospheric phenomena in accordance with their "realistic" positions near footpoints of geomagnetic field lines. An attempt is made to interpret some results from the PACE radar experiment using the calculated latitude ovals.
\end{abstract}

\section{Introduction}

It is well-known that the spherical coordinate system based on the Earth's dipole magnetic field was proposed and widely used to order a variety of phenomena and processes in nearEarth space. In that approach the $Z$ axis lies along the dipole axis of the Earth's internal magnetic field. This system is appropriate for use in the inner magnetosphere where the real geomagnetic field lines are very close to the dipole ones (CHAPMAN and BARTELS, 1940). To improve the ordering a number of eccentric dipole systems have been introduced because of the more intensive magnetic field over the Pacific than over the Atlantic area (SCHMIDT, 1934; COLE, 1963). The most recent ones have been used for a number of satellite studies including the high-latitude satellites DE-2 and VIKING. The chief advantage of the dipole and eccentric dipole systems is their spherical gridding and the simple transformation between magnetic and geographic coordinates.

A coordinate system useful for quantitative analysis of geophysical phenomena was introduced by HULTQUIST (1958) and was called the corrected geomagnetic coordinate system (CGM) after HAKURA (1965). By the definition, the magnetic field line is traced from the starting point up to the dipole equatorial plane using an internal field model only (International, 
IGRF, or Definitive, DGRF, Geomagnetic Reference Field models). The dipole field line is restored from the crossing point at the equatorial plane and the corresponding dipole coordinates are ascribed to the starting point on the Earth's surface or ionospheric altitude. It is obvious that there can be a significant difference between the calculated CGM latitude of a point and its real distance from the magnetic equator on the Earth's surface at low latitudes (GustafsSOn et al., 1992). Also, a magnetic field line starting from high-latitudes may cross the dipole equatorial plane so far from the Earth that it will be outside of the magnetosphere and therefore unrealistic. The geomagnetic field at high-latitudes is disturbed by external currents and ordering geophysical phenomena there in terms of the CGM system is deficient, since the real 3-dimensional structure of the magnetosphere is not taken into account.

GuSTAFSSON $(1970,1984)$ used the IGRF-1970 and IGRF-1980 models to calculate and tabulate the CGM coordinates relating to geographical grid. There are holes in the tables near the magnetic poles and calculations have not been done below $24^{\circ}$ geographic latitude in the northern hemisphere. BAKER and WING (1989) applied these tables to the southern hemisphere. They traced, first, all of the grid's points along field lines using the IGRF-1985 model with time derivatives updating the model to 1988. Then an expansion in terms of spherical harmonics was determined over the entire globe and for different ionospheric altitudes. By reversing the process, the inverse expansions relating the magnetic coordinates to geographic ones were also determined.

In the next section we describe an approach to build a new magnetic coordinate system which is time-dependent and based on the location of the minimum of the magnetic field, $B$ min, along a particular line of force. The approach is close to the so called Magnetic Apex Coordinates (VANZANDT et al., 1972) but the chief advantage of a new system is that it takes into account the external sources of magnetic field.

\section{Constant $B$-min (CBM) Magnetic Coordinate System}

The first problem to be solved here is to determine a definition of the magnetic equator line on the Earth's surface. The locus of $B$-min, as determined by the field model, determines a surface which is identified as the magnetic equator. However, a geomagnetic field line which starts from the Earth's surface very close to the equator may have several minima (2-3) along the line of force. This ambiguity is overcome by defining the magnetic equator by the straight line projection (along $\mathrm{R}_{\mathrm{E}}$ ) of the $B$-min point at a higher altitude where is only one minimum (GuSTAFSSON et al., 1992).

The CBM coordinates have latitudes defined from lines of constant $B$ on the warped 2dimensional magnetic equator surface. The procedure for determining the coordinates at a point on the Earth's surface or in space is a straightforward field line tracing from the starting point to the $B$-min point along the field line. The actual distance to $B$-min in unit of the Earth's radius, $R_{B}$, is then calculated from the relation:

$$
R_{B}=\left(H_{0} / B_{\min }\right)^{1 / 3}
$$

Here the equatorial field intensity $H_{0}=\left[\left(g_{1}\right)^{2}+\left(g_{1}\right)^{2}+\left(h_{1}{ }^{1}\right)^{2}\right]^{1 / 2}$ where $g_{1}{ }^{0}, g_{1}{ }^{1}, h_{1}{ }^{1}$ are the first order (dipole) IGRF coefficients. $R_{B}$ may be corrected for the fact that $B$-min is not at the dipole equator. The latitudes are defined as: 


$$
\cos \lambda=\left(R_{B}\right)^{-1 / 2}
$$

The latitude ovals in this coordinate system are projections along lines of force from the isolines of $B=$ constant at the $B$-min surface. This coordinate system has direct relevance to particles of $90^{\circ}$ pitch-angle which will drift in longitude along the magnetic equator on lines of $B=$ constant if no external magnetic and electric fields are present. The first invariant is zero at the magnetic equator and the magnetic field at mirror point is $B$-min. If for some reason the pitch-angle is changed into the loss cone, the CBM system can be used to project these particles along the field lines to the ionosphere.

\section{3. "Momentary" CBM Coordinate System for High-Latitudes}

Extending the above approach from low to high-latitudes we meet the second problem. External currents disturb the geomagnetic field and form the magnetopause in accordance with the model that is used. Magnetic field lines from very high-latitudes will be traced out of the realistic magnetosphere model box and will never reach the equatorial plane.

The following approach might be suggested. First, start to trace geomagnetic field lines from each degree of CGM latitude at $0^{\circ}$ in CGM longitude (which were calculated using only the internal magnetic field model) up to corresponding $B^{0}$-min values using both, internal and external, magnetic field models. These $B^{0}$-min values could be marked with the corresponding CGM latitudes. Then each point on the gridded geographical region (polar region or entire hemisphere) must be traced up to intersect the "real" magnetic equator surface using a field model with external sources (i.e. their $B$-min values must be calculated, gaps of calculated values will be obtained for the points which never cross the equatorial surface). These $B$-min values must be ascribed to the initial geographic points on the Earth's surface (or ionospheric altitude). Then the definite isoline of corresponding $B$-min value (which corresponds to the definite CGM latitude on the $0^{\circ} \mathrm{CGM}$ meridian) could be drawn on the Earth's surface as a quasi-latitude of the CBM coordinate system. It is an arbitrary agreement only to use the $0^{\circ}$ CGM meridian, any meridian can be selected as a basic one.

Figures 1 and 2 show the examples of the CBM quasi-latitudes over northern geographical frame for March 21, 1990. Iso- $B$-min contours are drawn in accordance with the above described rule. First column on each panel contains the number of the isoline on the figure which has definite $B$-min value (in nT, second column). The last one corresponds to the definite CGM latitude (third column) on the $0^{\circ} \mathrm{CGM}$ meridian. Contours above geographical latitude $80^{\circ}$ have not been drawn due to the lack of the $B$-min calculations for such high-latitudes. The corrected geomagnetic pole is marked by the cross on each figure. Calculations have been done by the original algorithm with the use Tsyganenko-1989 model of external sources (TsYGANENKO et al., 1987; TSYGANENKO, 1989) for four UT hours which are close to magnetic noon, midnight, dawn and dusk with respect to the north geomagnetic pole.

It is very easy to recognize from the figures the size and shape of polar cap for different UT and levels of activity. "Open" field lines form the shape of the polar cap very clearly. The cap has the "horse-collar" shape at noon and midnight, it becomes a quasi-circular at morning and evening. Some peculiar features appear on the dayside below the cusp region (closed contours on $70^{\circ}-72^{\circ}$ latitudes) and contours go very close to each other near magnetic midnight. The dayside features represent the entry layer and transition region between the cusp 

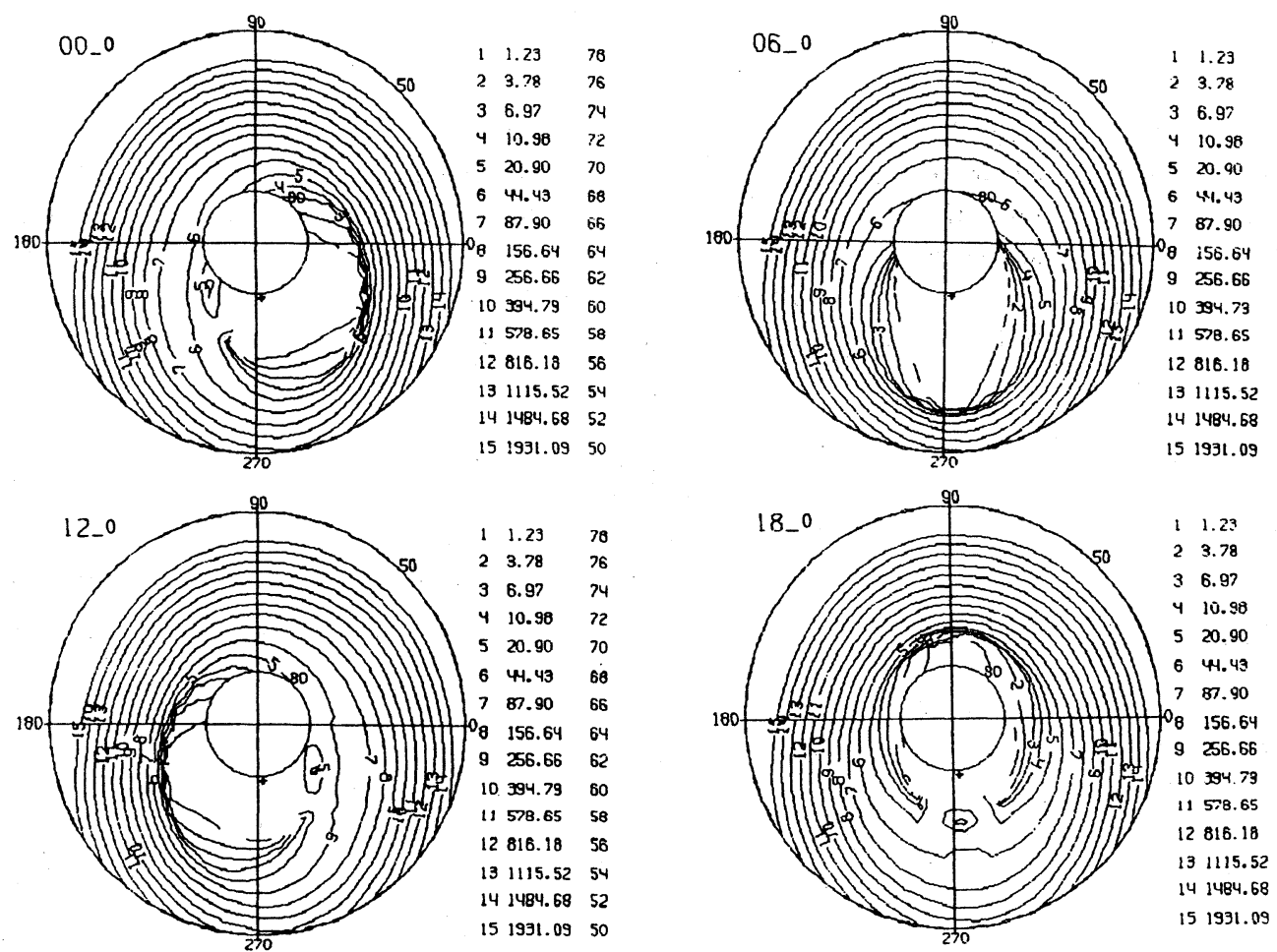

Fig. 1. Polar plots of the CBM latitudes over geographical coordinates in the northern hemisphere during quiet conditions $(K p=0)$ for the magnetic pole at dawn, noon, dusk and midnight $(0,6,12$ and $18 \mathrm{UT})$. CBM latitudes are marked by the numbers (first column) which correspond to $B$-min values (second columns) of the geomagnetic field lines started from the definite CGM latitudes on the $0^{\circ} \mathrm{CGM}$ meridian.

and sub-solar surface of the magnetopause. In the midnight sector we see the projection of the wide spatial region across the plasma sheet in the magnetospheric tail into a narrow belt of CBM latitudes at ionospheric altitudes. Some dashed lines above $76^{\circ} \mathrm{CGM}$ latitude (contour No. 1) appear due to formal restrictions on the model magnetospheric box ( $15 \mathrm{R}_{\mathrm{E}}$ along XGSM sunward and in the $Y-Z$ GSM plane, $70 \mathrm{R}_{\mathrm{E}}$ along XGSM tailward). Of course, this is a model and we cannot say that the real configuration of magnetosphere must be the same as described here.

These high-latitude contours (like No. 1) become more straight and cross the polar cap from midnight to dayside. They resemble the $\theta$-aurora which is observed mostly for quiet conditions. For $K p>3$ the polar cap expands considerably and the sub-cusp features extend along the latitude (throat-like features). Dashed contours come in accordance with general shape of the polar cap and look like "hooked" auroral arcs. In general, contours above the numbers 5 and $6\left(70^{\circ}-68^{\circ} \mathrm{CGM}\right.$ latitudes) for both quiet and disturbed conditions become quasi-circular and form an "oval-like" frame of the CBM coordinate system to order phenomena in auroral zone. But it is not a fixed system to describe phenomena for different UT and dates of seasons. We must re-calculate all contours for each moment of time under 

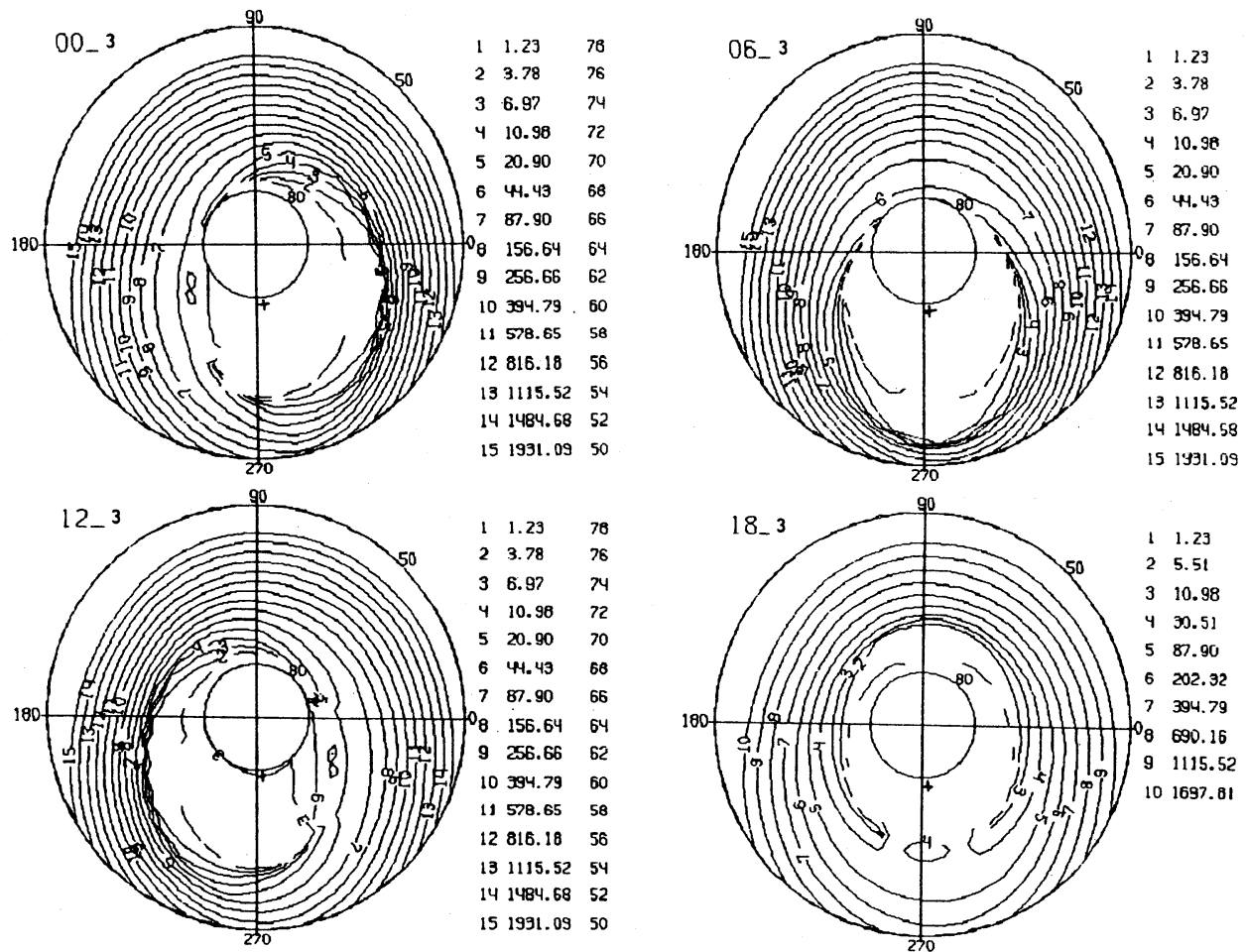

Fig. 2. The same as on Fig. 1 for disturbed conditions $(K p=3)$.

investigation. The CBM system is a geomagnetic coordinate system to analyse real events. It would be possible to order geophysical data for the equinox season in accordance with Figs. 1 and 2 by interpolating results between UT and $K p$ levels. But for the analysis of events or data during summer and winter seasons we must calculate new systems.

\section{Comparison the PACE Data in Two Coordinate Systems}

In order to see if the CBM coordinate system can in fact improve the ordering of geophysical data at high latitudes we present some results of the PACE radars (Polar AngloAmerican Conjugate Experiment; BAKER et al., 1989). In Figs. 3-5 the left panels on each plot show the Doppler velocities observed from the HF coherent radars at Goose Bay (GBA, $53^{\circ} \mathrm{N}$, $60^{\circ} \mathrm{W}$; top panel), Labrador, and Halley Station (HBA, $76^{\circ} \mathrm{S}, 27^{\circ} \mathrm{W}$, lower panel), Antarctica. These Doppler velocity maps are presented in PACE Geomagnetic Coordinates (BAKER and WING, 1989). We have chosen equatorial border of the scattering region as the reference lines for both observatories to compare their conjugacy. Each bin of the radar backscatter on these lines has geographical coordinates at the ionospheric altitude (about $300 \mathrm{~km}$ ) that were calculated from the radar measurements. We have examined the intensity of the backscattered signal to ensure that we are following the equatorial border of the region. Then we can trace each bin from one hemisphere to the other with the model described here. These original and 

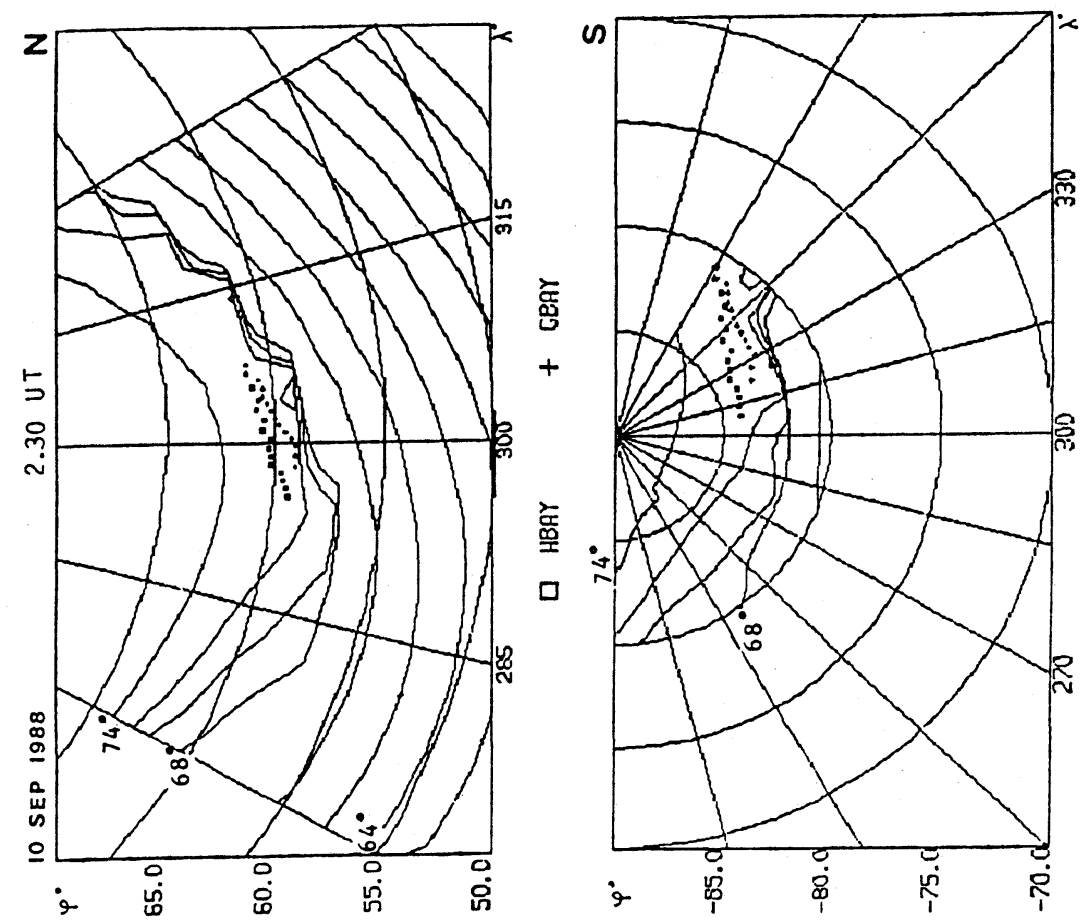

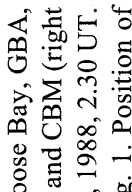

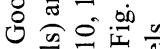

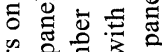

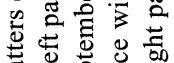

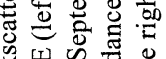

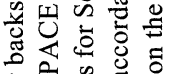

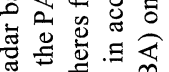

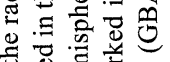

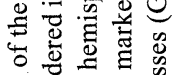

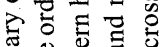

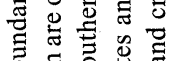

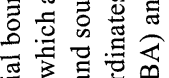

हึ

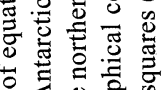

잉

영

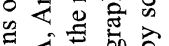

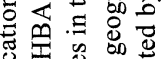

品

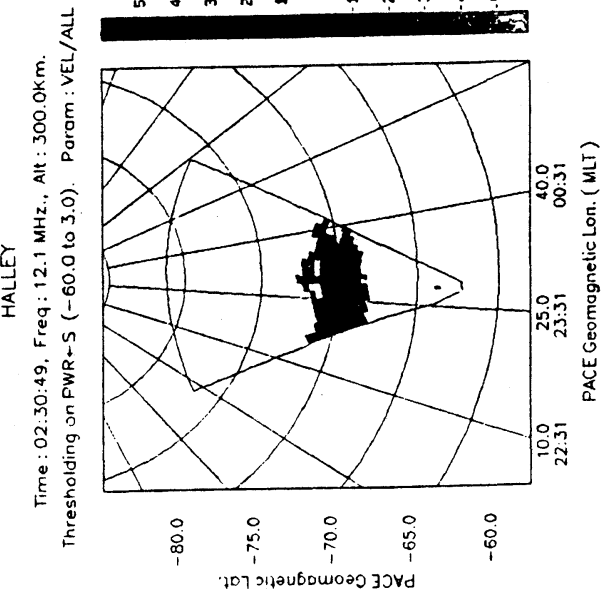

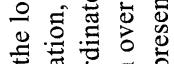

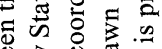

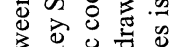

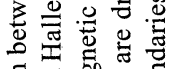

휴

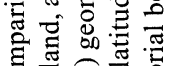

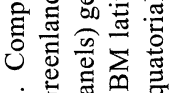
的芯嵒 宝 

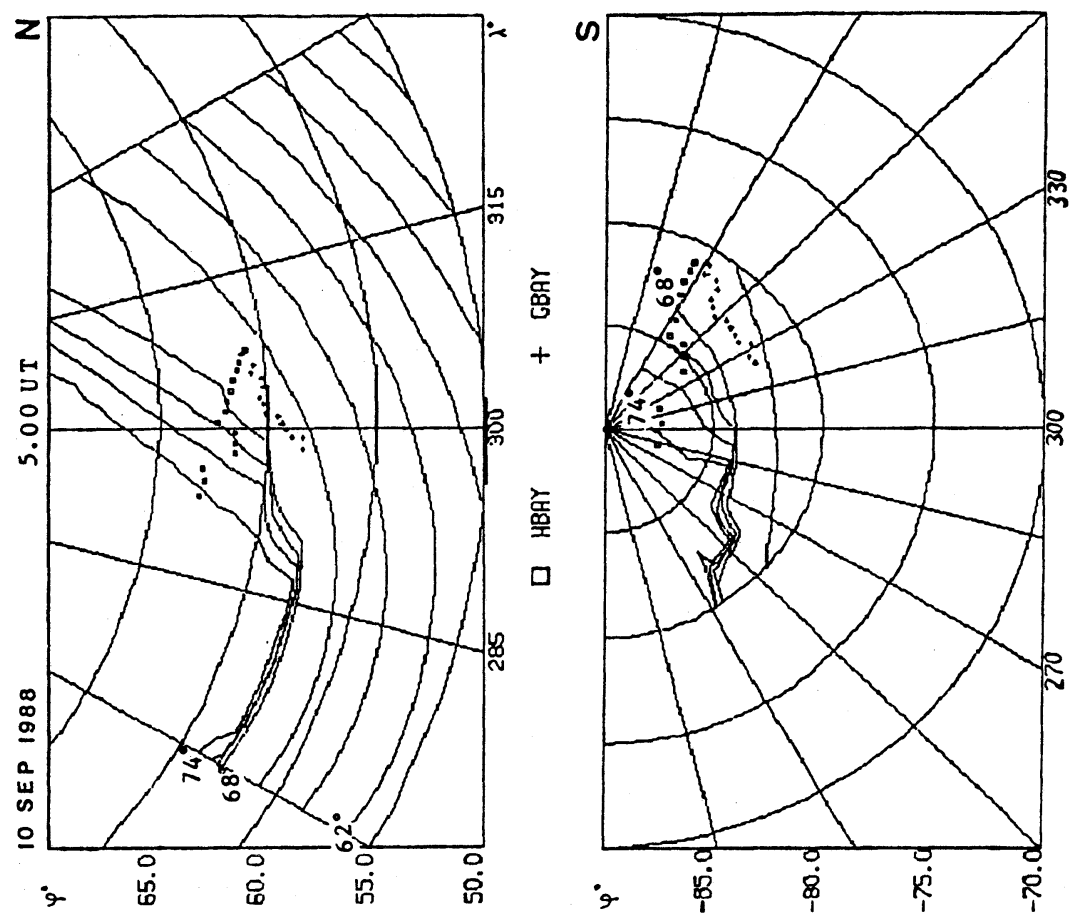

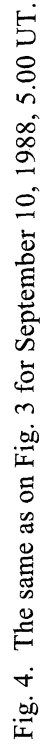
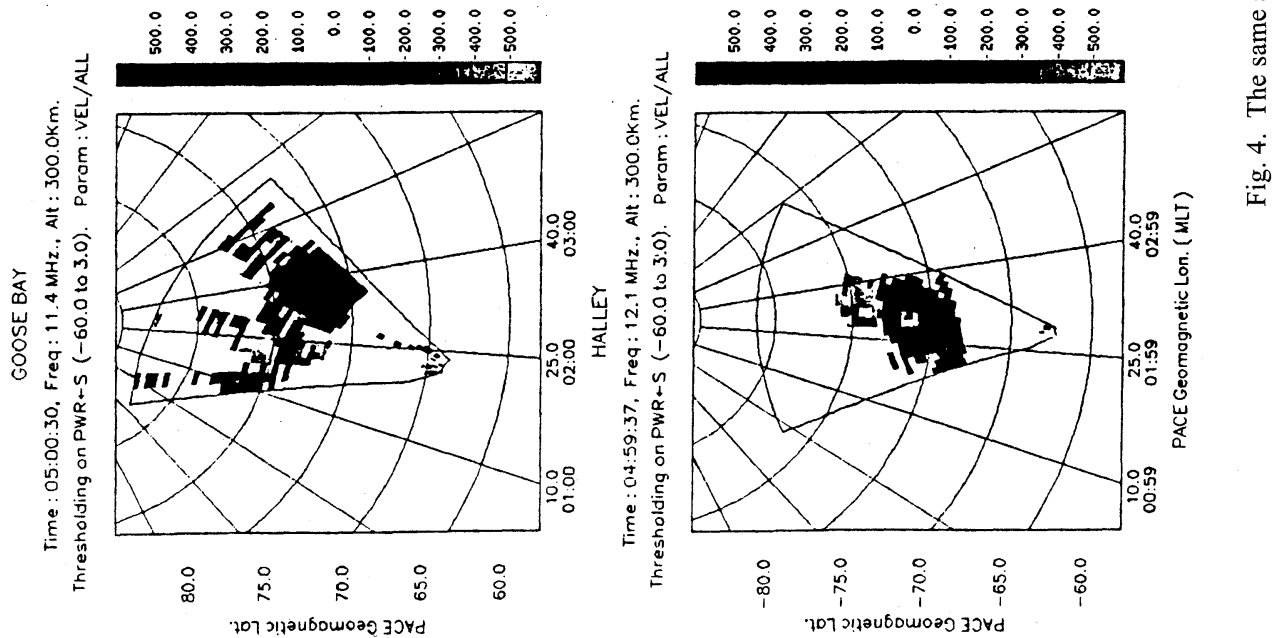

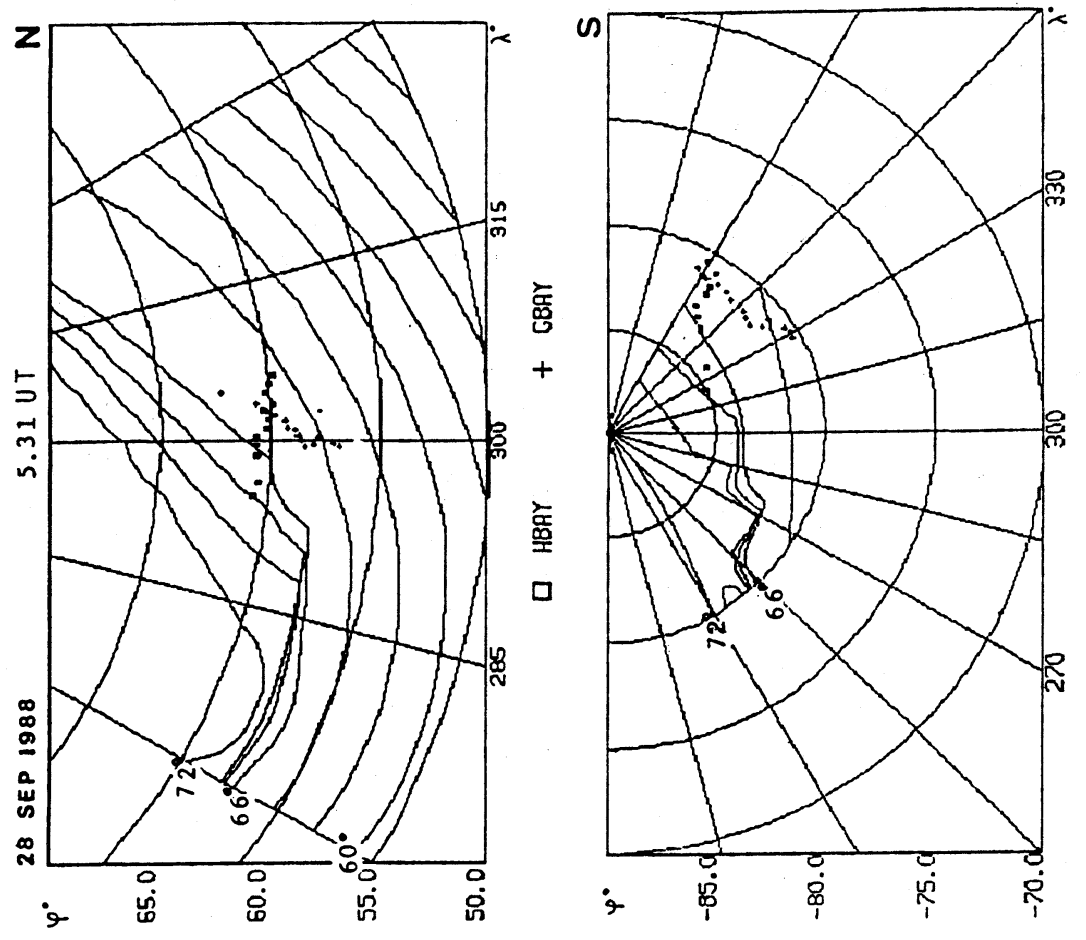

D
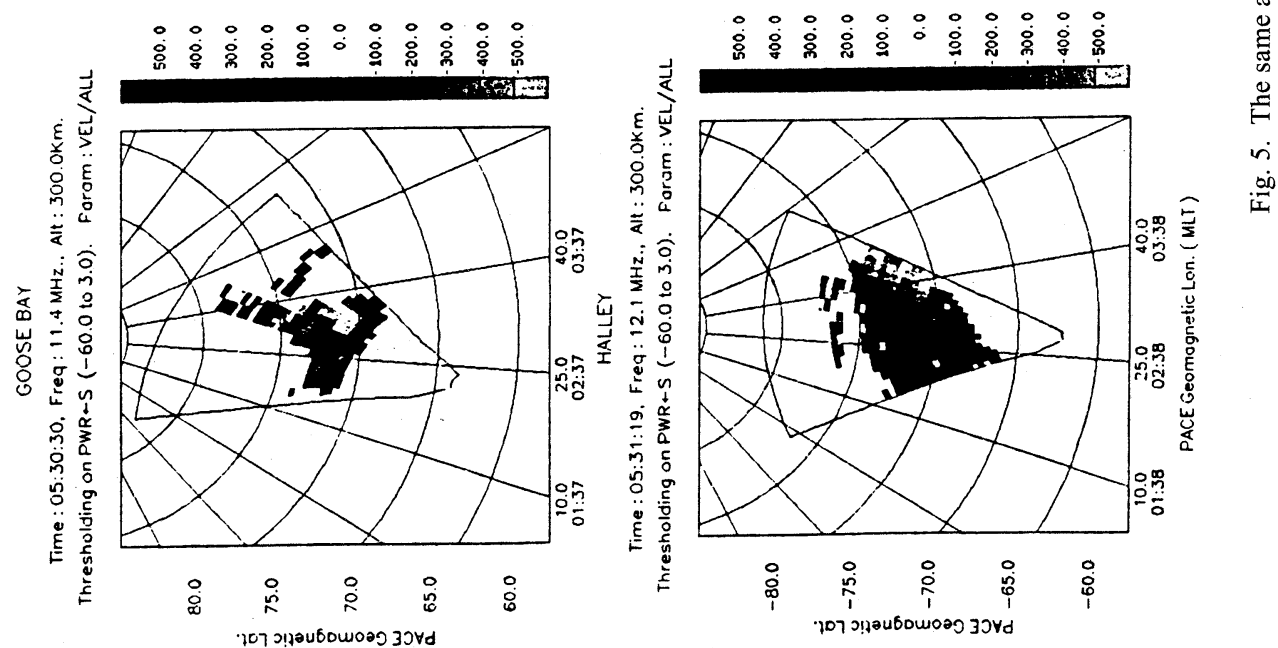
projected bins can then be overlapped with the new CBM coordinate system latitudes which are calculated for definite date, time and $K p$ (or $A E$ ) level.

Right panels of Figs. 3-5 show the CBM latitudes over geographical frame together with original and projected bins of equatorward boundary of the backscatter regions. One can see that the model conjugacy of data from GBA and HBA in the CBM system becomes much better for September 10, $2.30 \mathrm{UT}$, than for the PACE geomagnetic coordinates. The difference in latitudinal positions of the equatorial boundaries in the CBM system on Fig. 3 is less than $1^{\circ}$, compared to $2^{\circ}-3^{\circ}$ in the PACE system. But two other events (Sept. 10,5.00 UT, Fig. 4, and Sept. 28,5.31 UT, Fig. 5) show about the same level conjugacy of equatorial boundaries for both, CBM and PACE coordinate systems.

Analysing the positions of the backscatter boundaries in MLT we can say that improvement of conjugacy occurred near magnetic midnight. Two other events located a few hours dawnward show poor conjugacy for both the CBM and PACE systems.

\section{Discussion and Conclusions}

In order to understand the possible causes for the variation in the level of conjugacy in the CBM model we must consider how the backscatter observed in the ionosphere is connected to physical processes in the tail region. Let us assume that some patches of backscatter appear in the polar ionosphere due to some processes in the tail plasmasheet. Near midnight a spatial distribution of those processes is negligible in respect of their projection to the ionosphere along "real" magnetic field lines (in this case we are considering the model field line affected by external sources as a "real" line of force). Dawnward (duskward) the CBM latitudes diverge from each other and the spatial distribution of the patch sources becomes much more important. Of course, the location of ionospheric irregularities is affected by several processes in the $E$ - and $F$-regions (e.g., conductivity and electron concentration gradients). There is no a priori reason why these local phenomena should be the same in geomagnetically conjugate regions.

If we examine the PACE radar data in more detail there is one feature that may indicate another reason for the apparent lack of conjugacy seen in Figs. 4 and 5. First, when we examine the PACE data in Fig. 3, we note that the equatorial boundary is aligned along a line of constant magnetic latitude. In both, Figs. 4 and 5, however, the boundary shows a significant tilt with respect to the latitude lines. Also note that the direction of the tilt is opposite in the southern and northern hemispheres. These hemispherical asymmetries are characteristic of the IMF $B_{y}$ effects (GREENWALD et al., 1990). Since the Tsyganenko models are independent of $B_{y}$ effects, real variation in conjugate location due to $B_{y}$ effects cannot be successfully modelled with a coordinate system based on the current Tsyganenko magnetic field models. It should, however, be possible to include such effects into future magnetic field models and base a CBM coordinate system on such a model.

In conclusion we stress the importance of using real event studies in evaluating different magnetic coordinate systems as a way of ordering data. When a clear equatorial boundary of ionospheric backscatter is observed with both of the PACE radars, we expect that boundary to be a good marker of magnetically conjugate locations. It is clear that the PACE and CGM coordinate systems can produce significant latitudinal shifts of these conjugate locations. We have shown one instance where the CBM system clearly decreased the apparent latitudinal shift. In two other cases the CBM system did not improve the ordering of the data, but further 
development of the CBM system are possible, which would include the IMF $B_{y}$ effects. Such further development might greatly improve the ordering of geophysical data that a strongly affected by $B_{y}$ effects. We are currently examining other PACE events in order to determine how the present CBM coordinate system can be used in analyzing such data.

The authors would like to thank Principal Investigators of the PACE Project Dr. R. Greenwald (APL/JHU) and Dr. J. Dudeney (BAS) for providing the PACE data and helpful discussions.

\section{REFERENCES}

BAKER, K. B. and S. WING, A new magnetic coordinate system for conjugate studies at high-latitudes, J. Geophys. Res., 94, 9139-9143, 1989.

Baker, K. B., R. A. Greenwald, J. M. Ruohoniemi, J. R. Dudeney, M. Pinnock, N. Mattin, and J. M. Leonard, PACE-Polar Anglo-American Conjugate Experiment, EOS, 1989.

Chapman, S. and J. Bartels, Geomagnetism, 272 pp., Clarendon Press, Oxford, 1940.

Cole, K. D., Eccentric dipole co-ordinates, Aust. J. Phys., 16, 423, 1963.

Greenwald, R. A., K. B. Baker, J. M. Ruohoniemi, J. R. Dudeney, M. Pinnock, N. Mattin, J. M. Leonard, and R. P. LEPPING, Simultaneous conjugate observations of dynamic variations in high-latitude dayside convection due to changes in IMF $B_{y}, J$. Geophys. Res., 95, 8057-8072, 1990.

Gustafsson, G., A revised corrected geomagnetic coordinate system, Ark. Geofys., 5, 595-616, 1970.

GustafsSON, G., Corrected geomagnetic coordinates for Epoch 1980, in Magnetospheric Currents, Geophys. Monogr. Ser., Vol. 28, edited by T. Potemra, pp. 276-283, AGU, Washington, D.C., 1984.

Gustafsson, G., N. E. Papitashvili, and V. O. Papitashvili, A revised corrected geomagnetic coordinate system for Epochs 1985 and 1990, J. Atmos. Terr. Phys., 1992 (in press).

HAKURA, Y., Tables and maps of geomagnetic coordinates corrected by the higher spherical harmonic terms, Rep. Ionos. Space Res. Japan, 19, 121-157, 1965.

HultQuist, B., The geomagnetic field lines in higher approximation Ark. Geofys., 3, 63-69, 1958.

SCHMIDT, A., Der magnetische Mittelpunkt der Erde and seine Bedeutung, Gerlands Beitr. Geophys., 41, 346-356, 1934.

Tsyganenko, N. A., A magnetospheric magnetic field model with a warped tail current, Planet. Space Sci., 37, 5$20,1989$.

Tsyganenko, N. A., A. V. Usmanov, V. O. Papitashivili, N. E. Papitashvili, and V. A. Popov, Software for computations of the geomagnetic field and related coordinate systems, 58 p., Soviet Geophysical Committee, Moscow, 1987.

VAnZANDt, T. E., W. L. ClarK, and J. M. WARnock, Magnetic Apex coordinates: a magnetic coordinate system for the ionospheric $\mathrm{F}_{2}$ layer, J. Geophys. Res., 77, 2406-2411, 1972. 